\title{
Modeling and Measurement of 3D Deformation of Scoliotic Spine Using 2D X-ray Images *
}

\author{
Hao $\mathrm{Li}^{1}$, Wee Kheng Leow ${ }^{1}$, Chao-Hui Huang ${ }^{1}$, and Tet Sen Howe ${ }^{2}$ \\ 1 Dept. of Computer Science, National University of Singapore, Singapore \\ 2 Dept. of Orthopaedics, Singapore General Hospital, Singapore \\ \{lihao, leowwk, huangch\}@comp.nus.edu.sg, tshowe@sgh.com.sg
}

\begin{abstract}
Scoliosis causes deformations such as twisting and lateral bending of the spine. To correct scoliotic deformation, the extents of $3 D$ spinal deformation need to be measured. This paper studies the modeling and measurement of scoliotic spine based on $3 D$ curve model. Through modeling the spine as a $3 D$ Cosserat rod, the $3 D$ structure of a scoliotic spine can be recovered by obtaining the minimum potential energy registration of the rod to the scoliotic spine in the $x$-ray image. Test results show that it is possible to obtain accurate $3 D$ reconstruction using only the landmarks in a single view, provided that appropriate boundary conditions and elastic properties are included as constraints.
\end{abstract}

Key words: Scoliosis, spine, 3D reconstruction, modeling and measurement, deformation, Cosserat rod

\section{Introduction}

Scoliosis is a disease that causes deformations such as twisting and lateral bending of the spine. To correct scoliotic deformations by surgery and spinal fixation, the extents of $3 \mathrm{D}$ spinal deformations need to be measured $[1,2]$. In principle, these measurements can be made on the 3D model reconstructed from the patient's CT volume image of the spine. However, the radiation dosage of the patient in such a CT scan is too high. Therefore, x-ray imaging is currently the imaging technique of choice for the diagnosis and treatment of scoliosis. Multiple views of the patient's spine can be taken at the same time using biplanar radiography [3] or at different time using conventional radiography, with the patient turning to the side. Biplanar radiographic machines are bulky and inflexible. As they have limited use in clinical practice, they have been replaced by CT scanners. Therefore, conventional radiography is more commonly used for capturing x-ray images of scoliotic spines. A comparison of radiographic and CT methods for measuring vertebral rotation is given in [4].

There is a wide spectrum of existing works on the measurement of 3D scoliotic deformations based on x-ray images. In one extreme, a 3D curve is regarded as a simplified model of the spine, and it is registered to the spine in the x-ray

\footnotetext{
* This research is supported by A*STAR SERC 0521010103 (NUS R-252-000-319-305).
} 
image [5-7]. This approach is efficient but its accuracy is not guaranteed due to the simplicity of the model. In the other extreme, detailed patient-specific 3D models of vertebrae are reconstructed from biplanar radiography and then registered to the vertebrae in conventional radiographic images [8-10]. In theory, this approach should yield the most accurate measurements. However, it can be tedious and computationally very expensive.

From the anatomical point of view, the spine is a chain of rigid bodies (the vertebrae) that can rotate about their articulated joints within physical limits. Whereas a 3D curve may be too simplified a model for the spine, detailed patient-specific 3D models of vertebrae most likely contain too much redundant information for accurate measurement of scoliotic deformations. By determining the simplest model that produce accurate results, an efficient and less tedious approach for the measurement of scoliotic deformations can be identified. In this paper, we present an initial effort in determining the optimal model that balances accuracy and efficiency of measurement. Due to page limit constraint, this paper will focus on studying methods based on 3D curves. Methods based on detailed vertebra models will be studied in a follow-up paper.

\section{Related Work}

Existing work on the measurement of 3D deformations of scoliotic spine can be categorized according to the spine model used: (1) 3D curve and (2) detailed 3D model. For 3D curve-based approach, $[5,6]$ provides a GUI for the user to manually fit a 3D Bezier curve with 18 control points to the centerline of the spine in biplanar x-ray images. The fitted 3D curve is then used to perform Lenke and King classification of the type of scoliotic deformities [11,12]. In [7], the user manually fits a 3D Bezier curve with 6 control points to the centerline of the spine in biplanar x-ray images. In addition, the user identifies landmarks on key vertebrae in the images, and the algorithm interpolates the landmarks of other vertebrae based on the fitted 3D curve. The landmarks of the vertebrae are then used to compute the 3D positions and orientations of the vertebrae. These methods are computationally efficient but they require a lot of user inputs and interactions. Their accuracy depends on the expertise of the user.

For detailed model-based approach, the method of $[8,9]$ first extracts the centerline of the spine based on the segmentation algorithm of [13], which is used to compute the global positions of the vertebrae in the image. Then, it locally deforms a statistical vertebra model to register to the edges of individual vertebra in the x-ray image, subject to the constraint that the vertebra models form a smooth spine model. The statistical model is learned from a set of training scoliotic vertebrae [14]. The registered vertebra models can then be used to measure scoliotic deformations. Novosad et al. [10] fits patient-specific vertebra models reconstructed from biplanar radiography. The models are registered to the manually identified landmarks of the vertebrae in a single lateral bending $\mathrm{x}$-ray image, subject to the constraint that the vertebra models form a smooth spine. In principle, this approach can be very accurate, with a reported mean 


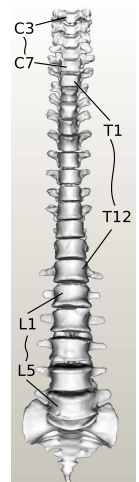

(a)

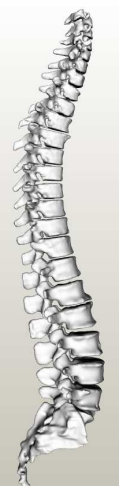

(b)

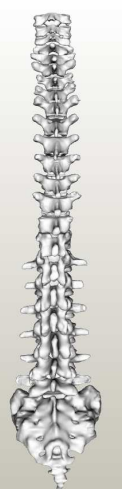

(c)

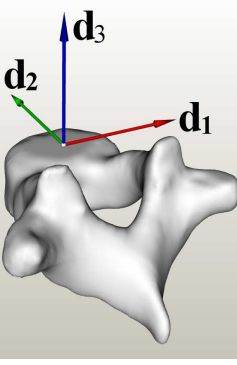

(d)

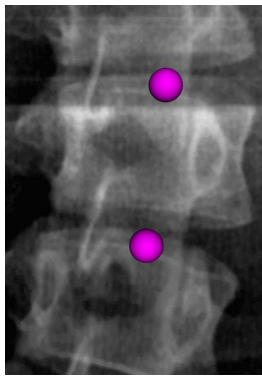

(e)

Fig. 1. Generic spine model. (a) Anterior view. (b) Lateral view. (c) Posterior view. (d) Vertebra with its directors. Triad indicates position and directors of vertebra. (e) Manually identified landmarks on the x-ray image. The landmarks are identified in the middle of the top end of each vertebra.

accuracy of about $1 \mathrm{~mm}$ on reconstructing a vertebra $[3,15]$. However, it is very tedious and computationally very expensive, especially for the reconstruction of patient-specific model of each vertebra of the spine.

\section{Modeling Scoliotic Deformations}

Our approach models a spine by a 3D curve with elastic properties called a Cosserat rod [16] (Sec. 3.1). The model is registered to the spine in x-ray images to recover the 3D structure of a scoliotic spine (Sec. 3.2). After registration, twisting and lateral bending of the model are computed and serve as the corresponding measurements of the patient's spine.

\subsection{D Spine Model Representation}

The spine is represented by a sequence of points $\mathbf{r}_{i}$ on the spinal centerline. Each point $\mathbf{r}_{i}$ corresponds to the mid-point on the top end of a vertebra (Fig. 1(d)). When projected to $2 \mathrm{D}$, this point remains in the middle of the top end of the vertebra, which is easy to identify (Fig. 1(e)).

Attached to each point $\mathbf{r}_{i}$ are the directors $\mathbf{d}_{i k}, k \in\{1,2,3\}$, which represent the $3 \mathrm{D}$ orientation of the vertebra (Fig. $1(\mathrm{~d})$ ). The director $\mathbf{d}_{i 3}$ is the tangent direction of the centerline at $\mathbf{r}_{i} . \mathbf{d}_{i 1}$ and $\mathbf{d}_{i 2}$ are the frontal and lateral directions of the vertebra that are always orthogonal to $\mathbf{d}_{i 3}$. The set of parameters $\left\{\mathbf{r}_{i}, \mathbf{d}_{i k}\right\}$ specifies the configuration of the model $M$.

According to the theory of Cosserat rod [16], the bending and twisting of the model can be defined based on the linear strain vector $\boldsymbol{v}_{i}$ and angular strain vector $\boldsymbol{u}_{i}$ such that 


$$
\boldsymbol{v}_{i}=\partial_{s} \mathbf{r}_{i}, \quad \partial_{s} \mathbf{d}_{i k}=\boldsymbol{u}_{i} \times \mathbf{d}_{i k}, \quad k \in\{1,2,3\} .
$$

The strain vectors $\boldsymbol{u}_{i}$ and $\boldsymbol{v}_{i}$ can be resolved into three components by the directors $\mathbf{d}_{i k}$ to compute the strain variables $u_{i k}$ and $v_{i k}$ :

$$
u_{i k}=\boldsymbol{u}_{i} \cdot \mathbf{d}_{i k}, \quad v_{i k}=\boldsymbol{v}_{i} \cdot \mathbf{d}_{i k}, \quad k \in\{1,2,3\} .
$$

The components $u_{i 1}$ and $u_{i 2}$ are the curvatures along directors $\mathbf{d}_{i 1}$ and $\mathbf{d}_{i 2}$. They measure the postero-anterior bending and lateral bending of the vertebra. The component $u_{i 3}$ measures twisting, whereas $v_{i 1}$ and $v_{i 2}$ measure shear, and $v_{i 3}$ measures stretching.

\subsection{D-2D Non-Rigid Registration}

The most common radiographic imaging technique in clinical practice captures the postero-anterior and lateral views of a patient's spine at different time. It is humanly impossible for the patient to keep exactly the same posture during the two captures. To accommodate such clinical practice, landmarks $\mathbf{m}_{i}$ are manually placed only on the vertebrae in the postero-anterior view.

The directors $\mathbf{d}_{i k}$ of the first and last vertebrae derived from the posteroanterior and lateral views serve as the boundary conditions. In Section 4, we will show that these data from the two views are sufficient to yield an accurate reconstruction of the 3D model of the patient's spine.

Non-rigid registration is performed by determining the configuration $\left\{\mathbf{r}_{i}, \mathbf{d}_{i k}\right\}$ of the model $M$ that minimizes the cost function:

$$
E=E_{f}+E_{I}
$$

subject to the boundary conditions. Quasi-Newton algorithm [17] is applied to optimize the cost function.

The term $E_{f}$ is the $3 \mathrm{D}-2 \mathrm{D}$ registration error:

$$
E_{f}=\sum_{i \in L}\left\|\mathbf{P}\left(\mathbf{r}_{i}\right)-\mathbf{m}_{i}\right\|_{2}^{2},
$$

where $\mathbf{P}$ is the projection matrix that projects the $3 \mathrm{D}$ point $\mathbf{r}_{i}=\left(x_{i}, y_{i}, z_{i}\right)$ onto the image plane. For simplicity, we assume weak perspective projection of the $\mathrm{x}$-ray images and the postero-anterior image plane is parallel to the $x-y$ plane of the world coordinate frame in which the 3D spine model resides. Therefore, the image plane and the world coordinate frame are related by scaling $s$ and translation $\mathbf{T}$. The $3 \mathrm{D}-2 \mathrm{D}$ registration error is thus:

$$
E_{f}=\sum_{i}\left\|s\left[\begin{array}{l}
x_{i} \\
y_{i}
\end{array}\right]+\mathbf{T}-\mathbf{m}_{i}\right\|_{2}^{2} .
$$

The potential energy $E_{I}$ that constrains the bending and twisting of the spine is defined according to the Cosserat rod theory:

$$
E_{I}=\sum_{i} \sum_{j=1}^{3}\left[\alpha_{i j}\left(u_{i j}-u_{i j}^{0}\right)^{2}+\beta_{i j}\left(v_{i j}-v_{i j}^{0}\right)^{2}\right],
$$


where $u_{i j}^{0}$ and $v_{i j}^{0}$ are the strain variables in the initial configuration of the spine. They represent the natural bending and twisting of a normal spine. The stiffness coefficients $\alpha_{i j}$ and $\beta_{i j}$ of the corresponding strains are dependent on the elastic properties and the geometrical properties of the model. The elastic properties, including the Young's modulus $Y$ and shear modulus $G$, are determined by applying Monte Carlo technique on a set of training data. In this way, the bending and twisting of the model will be consistent with those of actual spines.

\section{Experiments and Discussion}

Experiments were conducted to evaluate the proposed model, and to examine the necessary inputs for accurately reconstructing and measuring 3D scoliotic spine. Qualitative tests were performed on 30 pairs of real x-ray images (Sec. 4.1). For quantitative evaluation of the proposed model (Sec. 4.2), thirty sets of synthetic data were generated by manually adjusting the generic spine model to emulate different but realistic scoliotic spines in the real x-ray images.

Note that a spine has a total of 24 vertebrae. Medical assessment of scoliosis does not include the cervical vertebrae (Fig. 1, C3-C7). So, landmarks are not placed on them in the x-ray images. They are placed only on the thoracic vertebrae (T1-T12) and the lumbar vertebrae (L1-L5). In fact, some x-ray images do not contain cervical vertebrae.

\subsection{Tests on Real X-ray Images}

In the qualitative tests, for each test set, a pair of postero-anterior and lateral $\mathrm{x}$ ray images of scoliosis patient were provided. The landmarks on the vertebrae of the postero-anterior view were identified manually. The boundary conditions of the spine, i.e., the position $\mathbf{r}_{i}$ and directors $\mathbf{d}_{i k}$ of T1 and L5 were derived from the user specified points on both postero-anterior and lateral views. For each patient with a pair of $\mathrm{x}$-ray image, the number of input points was 21 in total. The proposed model took on the average 15 seconds to fit the landmarks in the $\mathrm{x}$-ray image, thus reconstructing the 3D patient-specific model of scoliotic spine. The average registration error (Eq. 4) is $0.228 \mathrm{~mm}$, which means the projection of the 3D model is very close to the landmarks in the postero-anterior view.

Three examples of the fitting results are illustrated in Figure 2, where the amount of lateral bending of the vertebrae are shaded in color. Note that although the lateral views provide only boundary conditions for the inputs, our reconstructed models can still fit the whole spines in the lateral views well. Therefore, our approach, with the trained elastic properties, is able to correctly model the bending and twisting of the actual spine. Since the lateral view was not scanned at the same time as the postero-anterior view, the posture of the patient may change between views. Thus, some of the reconstructed vertebra models do not align very well in the lateral views. On the other hand, since the landmarks in the postero-anterior views were given, the projections of the reconstructed spine models fit quite well with the postero-anterior x-ray images. 

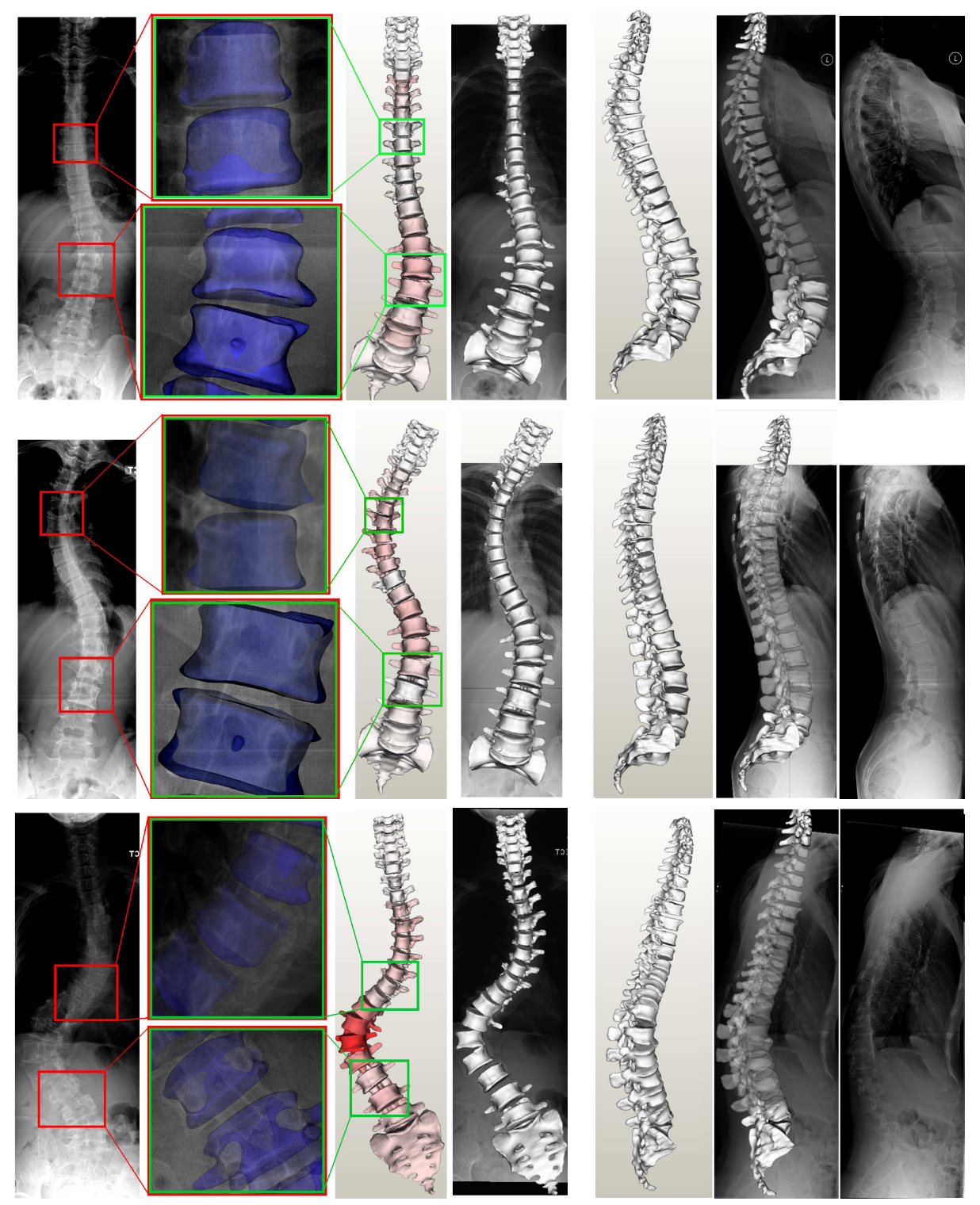

(a)

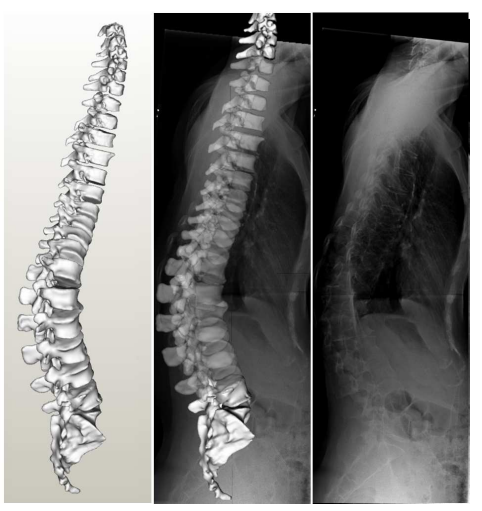

(b)

Fig. 2. 3D reconstruction results. Each row shows the results of a test set. (a) Posteroanterior view. (b) Lateral view. X-ray images and 3D models are overlapped and zoomed in to illustrate the fitting results. Zoomed-in 3D models in the overlapping images are set to transparent blue color. Vertebrae are shaded in red to illustrate the amount of lateral bending. 


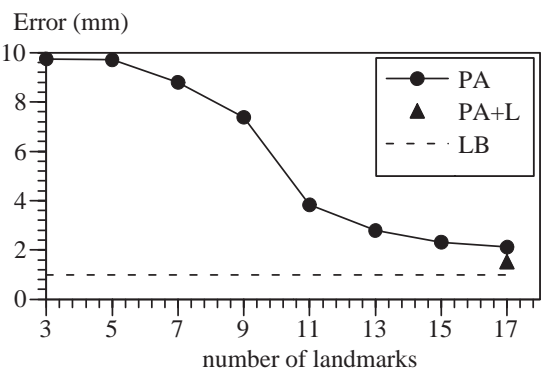

(a)

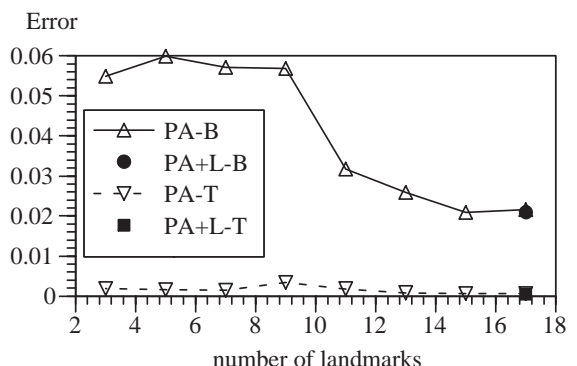

(b)

Fig. 3. Reconstruction errors for different number of input landmarks. (a) Mean fitting error of vertebra. PA: landmarks on postero-anterior view. PA+L: landmarks on PA and lateral view. LB: lower bound $[3,15]$. (b) Mean error of bending and twisting. -B: bending error. -T: twisting error.

\subsection{Quantitative Tests with Synthetic Data}

Thirty sets of synthetic data were generated for quantitative evaluation of the proposed model. For each test set, the ground truth spine model was obtained by manually adjusting the generic spine model to emulate a different but realistic scoliotic spine in the x-ray images. Eight test cases were performed with boundary conditions for T1 and L5 and varying number of postero-anterior landmarks (PA in Fig. 3(a)). For comparison, another test case was performed with 17 postero-anterior and 17 lateral landmarks (PA+L in Fig. 3(a)). For each test case, 3D reconstruction error, bending error, and twisting error averaged over 30 test sets were measured.

Figure 3(a) shows that the 3D reconstruction error decreases with increasing number of landmarks. For accurate reconstruction, e.g., error $\leq 2.3 \mathrm{~mm}, 15-17$ landmarks are required. With 17 postero-anterior landmarks, the reconstruction error is close to that obtained with 17 postero-anterior and 17 lateral landmarks. This indicates that the lateral landmarks are mostly redundant since the boundary conditions and elastic properties provide sufficient constraints to obtain accurate reconstruction. The reconstruction error of $2.114 \mathrm{~mm}$ for the PA-17 case is also close to the theoretical lower bound of $1 \mathrm{~mm}$ reported in $[3,15]$.

Figure $3(\mathrm{~b})$ shows that twisting error is not as strongly affected by the number of landmarks as the bending error. This is reasonable because the spine can bend more easily than it can twist. For accurate measurement of bending, sufficient number of landmarks, e.g., 15-17, are required.

\section{Conclusion}

This paper studies the modeling and measurement of scoliotic spine based on 3D curve model. The proposed method models a scoliotic spine as a 3D Cosserat rod. The reconstruction of the $3 \mathrm{D}$ scoliotic spine is achieved by obtaining the 
minimum potential energy registration of the rod to the scoliotic spine in the $\mathrm{x}$-ray image. Experimental results show that it is possible to obtain accurate 3D reconstruction using only the landmarks in a simple view, provided that appropriate boundary conditions and elastic properties are included as constraints.

\section{References}

1. Bernhardt, M., Bridwell, K.H.: Segmental analysis of the sagittal plane alignment of the normal thoracic and lumbar spines and thoracolumbar junction. In: 23rd Annual Meeting of the Scoliosis Research Society. (1988)

2. Panjabi, M., White, A.A.: A mathematical approach for three-dimensional analysis of the mechanics of the spine. Journal of Biomechanics 4 (1971) 203-211

3. Dumas, R., Aissaoui, R., Mitton, D., de Guise, J.: Personalized body segment parameters from biplanar low-dose radiography. IEEE Trans. Biomed. Eng. 52(10) (2005) 1756-1763

4. Lam, G.C., Hill, D.L., Le, L.H., Raso, J.V., Lou, E.H.: Vertebral rotation measurement: A summary and comparison of common radiographic and CT methods. Scoliosis 3 (2008) 16-25

5. Lin, H., Sucato, D.: Identification of Lenke spine deformity classification by simplified 3D spine model. In: Proc. Int. Conf. IEEE/EMBS. (2004) 3144-3146

6. Lin, H.: Identification of spinal deformity classification with total curvature analysis and artificial neural network. IEEE Trans. Biomed. Eng. 55(1) (2008) 376-382

7. Verdonck, B., Nijlunsing, R., Gerritsen, F.A.: Computer assisted quantitative analysis of deformities of the human spine. In: Proc. MICCAI. (1998) 822-831

8. Benameur, S., Mignotte, M., Parent, S., Labelle, H., Skalli, W., de Cuise, J.: 3D/2D registration and segmentation of scoliotic vertebrae using statistical models. Computerized Medical Imaging and Graphics 27 (2003) 321-337

9. Benameur, S., Mignotte, M., Labelle, H., Guise, J.A.D.: A hierarchical statistical modeling approach for the unsupervised 3-D biplanar reconstruction of the scoliotic spine. IEEE Trans. Biomed. Eng. 52(12) (2005) 2041-2057

10. Novosad, J., Cheriet, F., Petit, Y., Labelle, H.: Three-dimensional (3-D) reconstruction of the spine from a single $\mathrm{x}$-ray image and prior vertebra models. IEEE Trans. Biomed. Eng. 51(9) (2004) 1628-1639

11. Lenke, L.G.: Adolescent idiopathic scoliosis. The Journal of Bone and Joint Surgery 83 (2001) 1169-1181

12. King, H.A., Moe, J.H., Bradford, D.S.: The selection of fusion levels in thoracic idiopathic scoliosis. The Journal of Bone and Joint Surgery 65 (1983) 1302-1313

13. Kauffmann, C., de Guise, J.A.: Digital radiography segmentation of scoliotic vertebral body using deformable models. In: Proc. SPIE. (1997)

14. Benameur, S., Mignotte, M., Parent, S., Labelle, H., Skalli, W., de Guise, J.A.: 3D biplanar reconstruction of scoliotic vertebrae using statistical models. In: Proc. CVPR. (2001) 577-582

15. Mitulescu, A., Semaan, I., Guise, J.A.D., Leborgne1, P., Adamsbaum, C., Skalli1, W.: Validation of the non-stereo corresponding points stereoradiographic 3d reconstruction technique. Med. Biol. Eng. Comput. 39 (2001) 152-158

16. Antman, S.S.: Nonlinear Problems of Elasticity. Springer-Verlag (1995)

17. Press, W.H., Teukolsky, S.A., Vetterling, W.T., Flannery, B.P.: Numerical Recipes in C++: The Art of Scientific Computing. Cambridge University Press (2002) 